\title{
An Advanced Commercial Contact Center Based on Cloud Computing
}

\author{
Li Pengyu, Chen Xin, Zhang Guoping, Zhang Boju, and Huang Daochao
}

\begin{abstract}
With the rapid development of cloud computing and information technology, traditional contact center no longer satisfies the company's growth. Meanwhile, the new concept which focused on the needs of customers and services requires a much advanced contact center. Based on the technologies of cloud computing, Next Generation Network (NGN) and distributed technology, we first discusses the architecture of our advanced contact center, and then proposes the logic relationship of communication entities as well as a further design of centralized prototype for multimedia services and standard Session Initiation Protocol (SIP). So as to support typical calling process services, our approach includes two related examples; also, we design a typical signaling process for different services among involved network entities. Finally, we analyze the features and deployment applications of this novel cloud contact center.
\end{abstract}

Index Terms - Contact center, cloud computing, next generation network, multimedia.

\section{INTRODUCTION}

With the rapid development of IP communication, Internet of Things (IOT) and cloud computing technology, contact center has developed from traditional contact center to the new marketing center [1,2]. Contact center enables companies to significantly improve customer satisfaction by mainly focusing on object customers' business requirements, searching valuable customers, protesting investment and reducing their operating costs by themselves. In a word, contact center has become one of the crucial competitive strengths for these companies which are beneficial from deploying an enhanced contact center.

Traditional contact center has experienced some different periods such as circuit exchange, sound mixture, PBX-ACD, CTI (Computer Technology Integration) and so on $[3,4]$. These technologies suffered from a number of problems such as lacking service ability, scattered data source, hard to operate and apply. The problems mentioned above seriously affect modern companies' service quality and operating efficiency. With the spread of cloud computing, the traditional contact center is gradually replaced by the novel integrated and virtual cloud contact center. Based on the existing cloud computing and virtualization technologies, cloud contact center can easily utilize the development of virtualization mechanism, resource integration, and centralized management. Besides, cloud contact center provides standard data

Manuscript received May 22, 2012; revised June 15, 2012.

Li Pengyu, Zhang Guoping and Zhang Boju are with Beijing Huiyuan Jiyun Information Technology Corporation, Beijing, China (e-mail: maxlipengyu@gmail.com).

Chen Xin is with North Carolina State University, Raleigh, USA

Huang Daochao is with Beijing Jiaotong University, Beijing, China. interfaces and structures to receive different applications, such as voice, video, fax, Email, social media and web application, which can form an integrated platform for all medias with bidirectional input and output services [5]-[6]. Comparing with the traditional contact center, cloud contact center has advantages of environmental protection and high capacity, which provide high quality of communication ways, convenient business, smart agent and self-assistant services for customers.

The rest of the paper is organized as follows. Section 2 introduces contact center system architecture and logic relation among different communication entities; Section 3 describes two kinds of typical calling process of cloud contact center; while Section 4 analyses the features of cloud contact center and methods of deploy application; Section 5 summarizes this paper.

\section{Cloud Contact Center Architecture}

\section{A. Contact Center System architecture based on Cloud Computing}

In the traditional contact center, key functions such as call control, business application and data exchange are gathered into one facility. Yet the contact center based on cloud computing adopts the centralized and integrated design idea with the help of virtual network, integrating resource and centralized management. By this means, we can build a four-layer system which contains IAAS (Infrastructure as a Service), PAAS (Platform as a Service), SAAS (Software as a Service) and Enterprise Application. The designed system architecture is shown in Fig. 1.

1) IAAS Layer. IAAS layer provides an elastic computing resource pool and virtual computing capabilities, which can offer dynamic resources and make a quick deployment according to customer requirements.

2) PAAS Layer. PAAS layer provides a high reliable contact center resource with unified control and data center, which forms a standard interface for different applications. Meanwhile, business and internet gateway are able to provide free and open application platform for developers and other applications.

3) SAAS Layer. SAAS layer delivers sufficient business cloud resources based on voice, agent and data resource. All customers are able to work with cloud services by the cloud platform through SAAS layer.

4) Enterprise Application Layer. With the help of application interface, data resource and servers, the cloud platform can offer many business methods, valuable services and interrelated API interfaces to users. 


\section{B. Cloud Contact Center Core Communication Entity}

The platform consists of accessible cloud, IP cloud, computing cloud and data resource, the most important one is computing cloud. The whole platform of cloud contact center composes by four parts such as SoftACD pool, CTI pool,
IVR pool and Agent pool. All access medias can be managed by SoftACD pool and CTI pool to line up and route $[7,8,9]$. The logic relationship of these communication entities is depicted in Fig. 2.

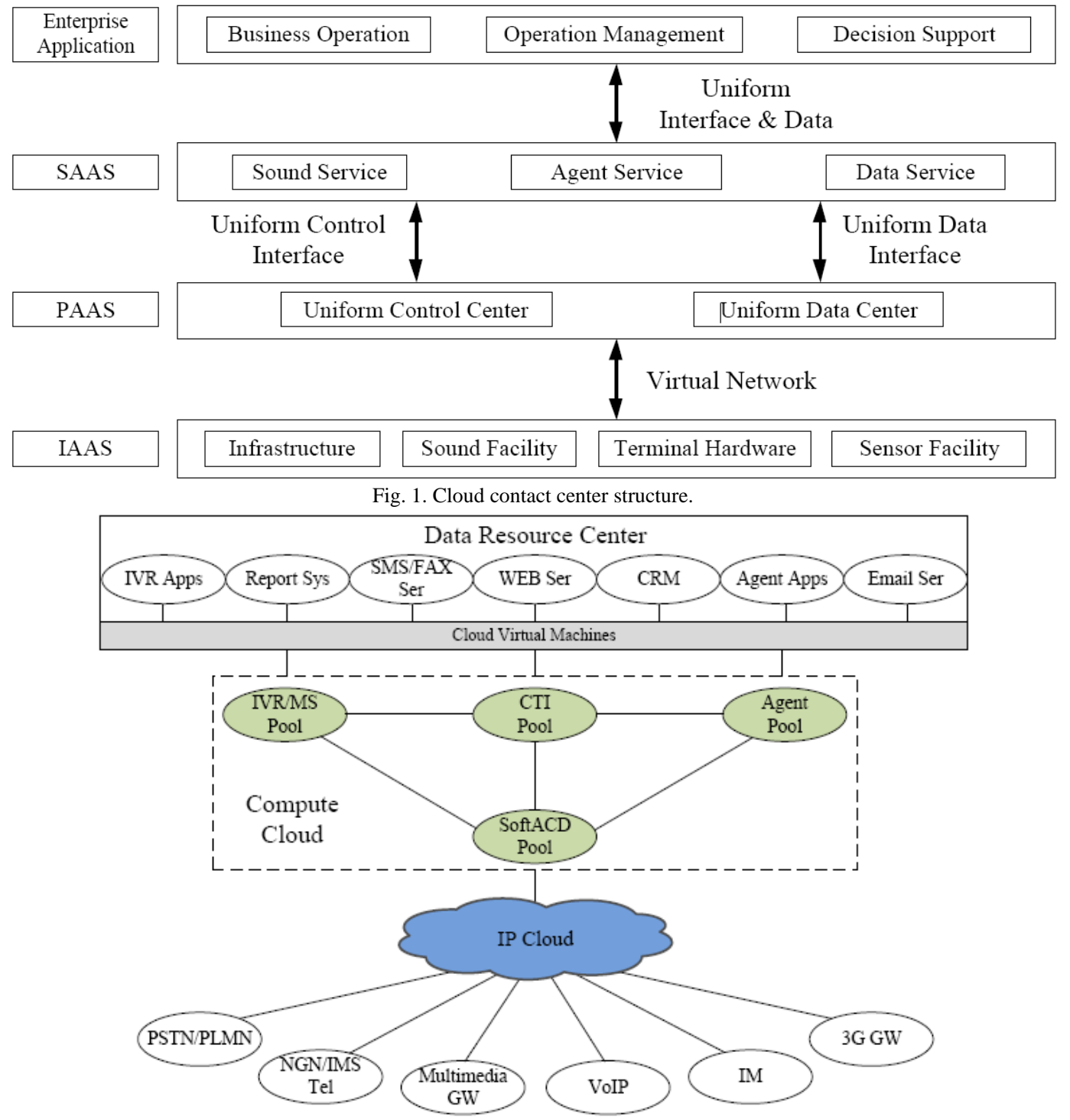

Fig. 2. The logic structure of cloud platform.

Figure 2 shows that, SoftACD pool controls the access of all voice, video and other media. CTI pool make a unique routing and queuing for all call events and manage the system's resource for other events. IVR pool provide selfassistant service voice, video and other business media resources. Agent pool supply all kinds of personalized services by the ways of call-in and call-out. Application servers include many useful management tools for administrators and users.

1) Integrated Communication Platform based on Cloud Computing

Cloud contact center use standard SIP protocol to control signals and calls. All kinds of media terminals and heterogeneous networks reach unique access via cloud platform's data and control center. All access information was gathered by SoftACD pool to queue and distribute. Signals arriving SoftACD pool have no relationship with SIP dialog's media information; they do not have any differences among terminals and networks in queuing system, so the cloud plat- form can receive many kinds of signals from media terminals. Each application has a sole interface number. So the service enable middleware can shield lower layer's networks and protocols. After all, cloud contact center can build up a unique communication platform for different terminals and users.

2) Standard Business System based on SIP

Cloud platform uses standard SIP protocol following NGN network architecture principles. Specialized at open and fusion, the platform can offer the unified service based on IP cloud to construct a standard SIP service system. The system owns integrated network feature and structure, and provides with flexible abilities of access and communication for many multi-medias.

Based on IP cloud and cloud computing, the system can complete signaling controlling and transiting of voice carrying signaling. Also, it has features of flexible control and strong scalability. With a unified control center and data center, cloud platform achieves the separation of business 
and control, control and carrying. And meanwhile, the platform reaches to independence between business development and call control, call control and session media. So the control center only handles the session control and the necessary business trigger. And the data center directly processes media session information. As a result, at different levels, the system will form functional independent communication entities. The communication can be realized between the communication entity and each level by standardized communication interface.

\section{The Design of TypicAl CALling Process IN Cloud CONTACT CENTER}

From the perspective of business applications , the typical cloud contact center calling process can be divided into two major parts such as call-in and call-out business. Call-in business is that contact center receives user's calling to provide business service. Call-out business is that contact center agents initiatively call users to spread products and services.

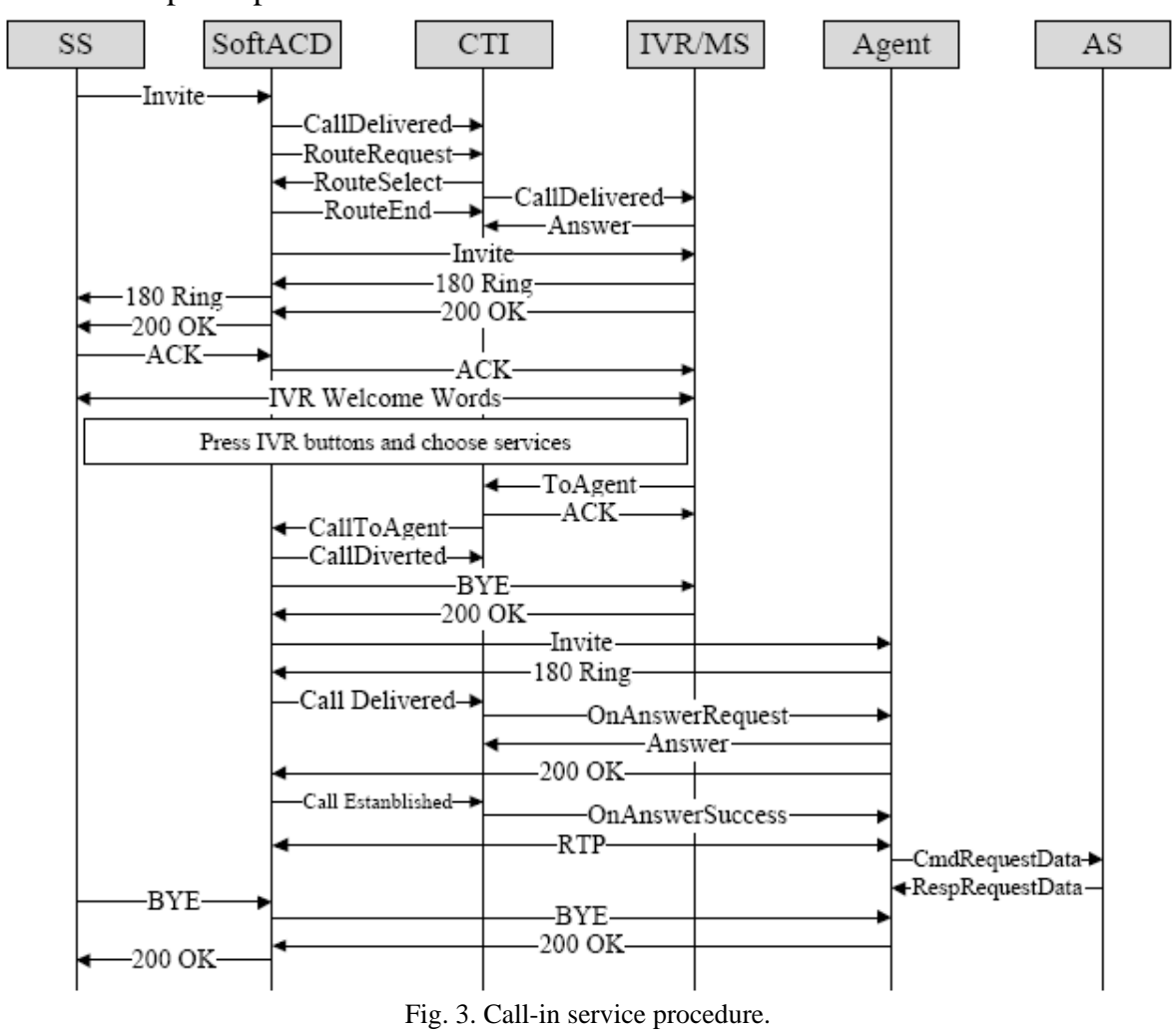

As shown in Figure.3, user's call information will be sent into the cloud platform through IP cloud, and form standard interface information (SIS) in the cloud platform. Firstly, SIS sends an invite request to SoftACD pool, when SoftACD pool receive call request, they send CallDeliverd event and RouteRequest route information to CTI pools for alerting call arriving. Based on route strategy, CTI pools reply RouteRequest information to SoftACD pool, and then the coming call turns to IVR pools, CTI pools send CallDelivered event to IVR pools for alerting the system. When IVR pools receive call signals, it will broadcast IVR leading information to customers. While listening the IVR information, the customer can choose one kind of service by dialing choosing button. If one chooses assistance, IVR will send assistance request to CTI pools, CTI pools start to obtain
We will do some analysis and research on call-in and callout business's signal communication procedure with the example of calling process.

\section{A. Call-in Service Process}

When PSTN, NGN, 3G, smart network and other multimedia terminals call into the contact center, incoming information goes through IP cloud transferring control signal and media data to the cloud platform of unified control and data center, then the platform organizes the whole calling process among logical communication entities. CTI pools manage and configure all system resources to provide intelligent route strategy for user's calling process. SoftACD pool send calling information and route request to CTI pools; CTI pools get route information and send back to SoftACD pool. With the direction of the route information, SoftACD pool connect to the destination agent; the whole calling process will be build up. The call-in service process is shown in Fig. 3.

free agent resource. If all agents are busy now, the custom will continue to share system music. Then CTI pools send CallToAgent information to SoftACD pool, SoftACD pool will connect to the free agent, when the agent answers, the calling procedure will be build up between user and agent. In this case, agent can visit CRM system or other application servers to get some information and service based on user's requirement.

\section{B. Call-out Service Process}

The call-out service is the procedure that agents initiate calls to users. First, Agents will call CTI pools, and then SoftACD pool and CTI pools decide together to establish the call process between SoftACD pool and Agents, SoftACD pool and users. Finally, SoftACD pool establishes the 
communication between users and agents. The procedure is shown in Fig. 4.

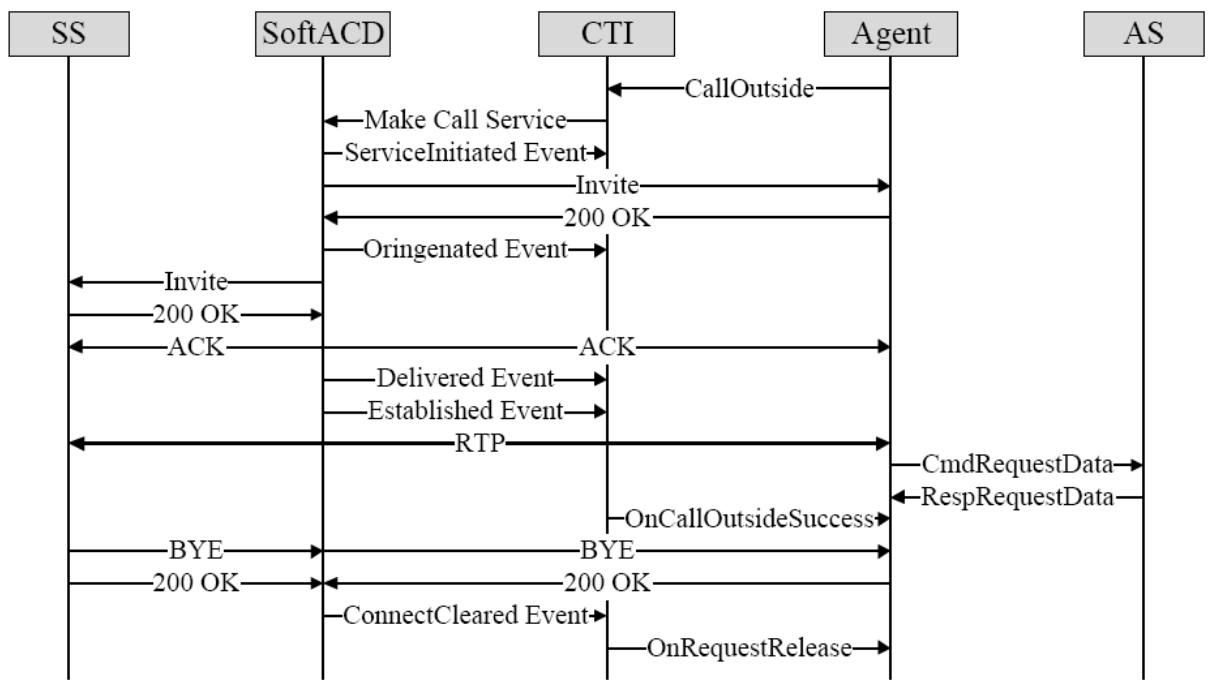

Fig. 4. Call-out service procedure.

Shown in Fig. 4, agent sends an invite request for CTI pools, CTI pools receive request information and inform SoftACD pool to start initial service. SoftACD pool reply initial information to CTI pools and send an invite request to agent, receiving the response from the agent, and then send a connected event to CTI pools. After that, SoftACD pool send an invite request to user's terminal, when it get user's response, SoftACD pool replies ACK to user's terminals and CTI pools, in the same time, it sends Delivered Event and Established Event to CTI pools. Then, the call will be build up between agents and users. Agent can make use of CRM or application server to provide some service to user. When the whole calling ends, user hangs up and sends BYE information to SoftACD pool, SoftACD pool send the BYE to agent and receive $200 \mathrm{OK}$ information from the agent. At the same time, SoftACD pool send ConnectCleared to disconnect with CTI pools, CTI pools repsponse OnRequestRelease event. The whole call process will be ended.

\section{Application of Cloud Contact Center}

With an integration and virtualization design idea, cloud contact center has built a unified cloud platform based on IP cloud and computing cloud and the platform implements the interconnection by the standardized control and interactive data interfaces. The deployment of cloud contact center starts from data centers, which forms an integrated entity for external services via computing cloud and IP cloud, while the management center, business center, media center and pew center rely on the data center. The cloud contact center system supports the traditional distributed layout and centralized management, implementing a cross-regional and crossboundary unified contact center. By the technologies of convergence and virtualization, we can easily achieve a unique ultra-logical contact center in national and even global scale. It can also realize the effective integration of infrastructure and services of contact center. The applications of cloud contact center are shown in Fig. 5.

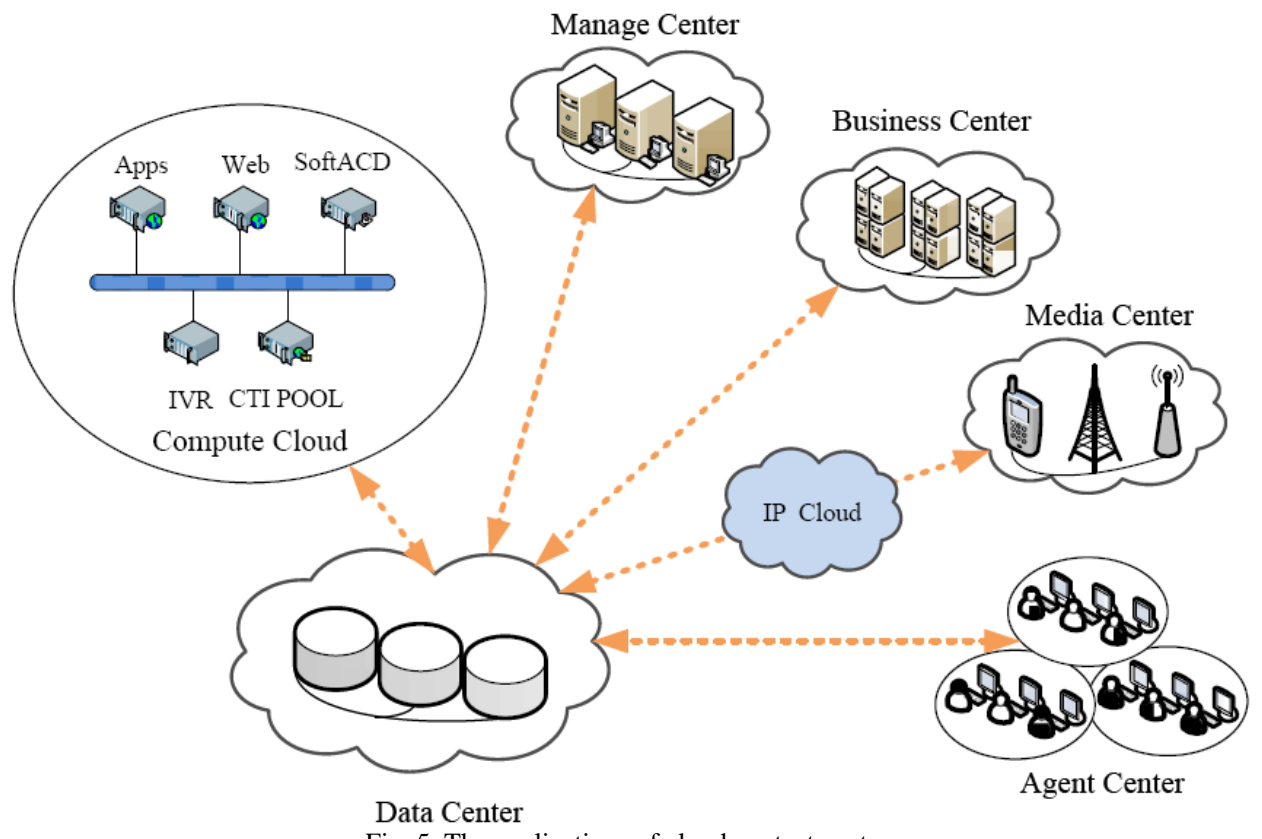

Fig. 5. The applications of cloud contact center. 


\section{A. Feature analysis of Cloud Contact Center}

Cloud contact center can provide required cloud services, efficient mobile cloud management, cloud collaboration via seamless communication and the green open cloudy agents. The cloud contact center can greatly reduce the cost for construction and maintenance of the contact center, shorten the construction time, and provide personalized services to meet customer's need, which will greatly enhance the customer satisfaction.

As shown in Figure.5, with the help of virtualization, distributed computing and cluster technology, several physically separated sets of contact center platform can compose shared scheduling pool (CTI Pools), which presents a set of high-capacity contact center platforms to upper applications, therefore, the upper applications do not care or manage the physical nodes and the deployment of business objects. Cloud platforms can achieve the resource virtualization by virtualizing into multiple independent logical contact centers with independent deployment of services and monitoring management.

Cloud contact management center adopts a web management platform based on B/S structure. As long as they can connect to the internet and establish a secure channel for data communication, the secure remote operation and maintenance management can be implemented at anytime and anywhere. In addition, by providing a rich graphical monitoring interface, the real-time system operation and maintenance status, quality of service can be conveniently monitored and managed, in the same time, the area traffic and the operational status of each network element can be take into statistics in time.

Cloud contact media center can manage kind of media's access. The media center not only supports common voice's access, but also support Email, Fax, short message, video and other media's access methods. Users can communicate with the agents by voice, picture, literature and other services, which can expand the content of commutation and boost the efficiency for all users.

Cloud contact business center can provide enhanced customized and individual businesses; also support distributed and centralized network functions. If one branch organism wants to expand the scale of agents, it can only apply more cloud application resource to update the whole contact center.

Nowadays, cloud agents make use of VoIP soft telephone terminal to achieve bidirectional voice and video communication. When soft telephone terminals are combined with Web browser, they form an online calling control system; the system will be very convenient to accomplish mobile agent and remote access.

\section{B. Deploy Applications of Cloud Contact Center}

For cloud contact center, call business center, management center, media center, date center and agent center can be deployed in centralized or diverted ways. The system can be adjusted to several of business modes, such as integrated access with integrated handling, integrated access with diverse handling, diverse access with integrated handling, diverse access with diverse handling, etc. In addition, special green data center satisfies users by achieving the object of integrated design, diverse deploy, expanding by requirements, and dynamic adjustments. So it is easy to implement green agent, home agent or remote access.

\section{CONCLUSIONS}

With the help of cloud computing, virtualization technology, integrated technology and IP cloud, the cloud contact center can provide an integrated service for all customers. Under this content, this paper studies the system architecture and logic relationship among key communication entities of cloud contact center. Then, based on the logical relationship between different entities, such as SoftACD pool, CTI pool, IVR pool and Agent pool, we design the business procedures of call-in and call-out in a typical calling. Finally, we analyze the features and discuss how to deploy applications of cloud contact center. Our research results are very useful to the deployment for applications of cloud contact center in the cloud computing environment.

\section{REFERENCES}

[1] Y. Zhao, "Research and Accomplishment of NGN Contact center based on Soft Switch Structure," ShangHai Jiaotong University, 2010

[2] K. Wang, "Design and Accomplishment of Distributed Contact center," Fudan University, 2008.

[3] Q. Zhao, C. Zhang, and R. Zuo, NGN technology based on Soft Switch and Application, Beijing: Post \& Telecom Press, 2009.

[4] H. Gui and B. Zhang, Soft Switch and NGN, Beijing: Post \& Telecom Press, 2009.

[5] H. Wu, Cloud computing core technology analysis, Beijing: Post \& Telecom Press, 2011.

[6] Petrunka et al., Internet Network Contact center, United States Patent: 6122364, Sep. 19, 2000.

[7] Uppaluru et al., Point-of-Presence Contact center Management System, United States Patent: 6011844, Jan. 4, 2000.

[8] Foladare et al., Virtual Contact center, United States Patent: 6049602, Apr. 4, 2000.

[9] Price. Multi-Tasking Web-Based Contact center, United States Patent: 6389132 B1, May.14, 2002.

[10] A. Bassamboo, J. M. Harrison, and A. Zeevi, Design and Control of a Large Contact center: Asymptotic Analysis of an LP-based Method, Submit June 4, 2004

[11] H. S. Saha, R. Roy, and M. M. Hussain, Capacity Planning and Dimensioning of an Agricultural Contact center. 